\title{
A Joint PIC/Decoding Algorithm for Convolutionally Coded CDMA Systems
}

\author{
Guoxiong $\mathrm{Xu}^{1}$ and Feng $\mathrm{Ji}^{2}$ \\ ${ }^{1}$ School of Electric and Information Engineer, Zhongyuan University of Technology, Zhengzhou, China \\ ${ }^{2}$ Library, Zhongyuan University of Technology, Zhengzhou, China
}

\begin{abstract}
This paper mainly explores parallel interference cancellation and decoding (PIC/decoding) algorithm for convolutionally coded DS-CDMA systems. A new joint PIC/decoding scheme is proposed and analylized, respectively. The proposed scheme is numerically simulated, and compared with other PIC/decoding algorithm over Rayleigh fading channel. From the simulations, the main conclusion can be drawn as : (1) like conventional PIC and decoding algorithms, the proposed algorithms exhibit better performance with the increase in the number of the interference cancellation (IC) stages, but up to three IC stages, the performance improvement is getting less obvious; (2) the proposed joint PIC/decoding acquires the performance close to the ideal joint PIC/decoding if the tentative decisions of coded symbols are linearly estimated by the sum of initial a priori log-likelihood rate (LLR) and updated a priori LLR, while a significant performance degradation will evitably occur if the tentative decisions of coded symbols are linearly computed by the updated $a$ priori LLR only. We also compare the proposed PIC/decoding with matched-filter (MF) receiver and conventional PIC/decoding algorithms, respectively. It is shown that the proposed joint PIC/decoding dominantly outperforms the MF receiver and conventional PIC/decodings in performance.
\end{abstract}

Keywords-DS-CDMA; interference cancellation; decoding; loglikelihood rate(LLR); simulation

\section{INTRODUCTION}

Arising from the nonideal crosscorrelation properties of the spreading signals, the interference among active users, i.e., multi-access interference (MAI), is the main factor restraining code-division multi-access (CDMA) systems from improving performance. Using the conventional matched-filted (MF) receiver to detect such CDMA signals will lead to unacceptable performance loss. Multi-user detection (MUD) [1-2], though sophisticated, can effectively suppress MAI and overcome the performance degradation of CDMA systems. Among these MUDs, one suboptimal, but relatively simple method is the parallel interference cancellation (PIC) [3-4]. In general, PIC is of a multi-stage structure.

To ensure the reliable data transmission, CDMA is generally combined with convolutional encoding, which is socalled convolutionally-coded CDMA. In convolutionally-coded CDMA systems, conventional PIC is classfied into two kinds depending on different combination methods of PIC and decoding. One is called separate PIC and decoding, which performs interference cancellation (IC) before decoding; while the other is called joint PIC and decoding, in which decoding and re-encoding are performed to obtain tentative values of coded symbols at each IC stage [5]. It is shown that the latter brings a significant performance gain with a higher computation complexity and a relatively long processing delay.

Modifying the joint PIC and decoding algorithm, this paper proposes a low-complexity joint parallel cancellation/decoding for convolutionally-coded CDMA systems.

\section{SYSTEM MODEL}

For simplicity, we explore a synchronous convolutionally coded CDMA system and the signals are transmitted over Rayleigh fading channel. Convolutionally encoding and BPSK modulation are performed for binary information bits. After being spread, the coded symbols are transmitted.

A convolutional encoder with constraint length $v$ and rate $1 / n$ is considered. Binary information bit $b_{k}(p)$ at the $p$-th bit interval for user $k$ is input to the corresponding encoder, the $q$ th coded symbol output is expressed as $d_{k, p}^{q},(q=1,2, \cdots, n)$, which can be rewritten as $d_{k}((p-1) n+q)$, i.e., $d_{k, p}^{q}=d_{k}((p-1) n+q)$.

Rayleigh fading channel is considered, and thus we can express channel complex transfer impulse response as

$$
\xi_{k}=\alpha_{k} \exp \left(j \beta_{k}\right)
$$

where $\left\{\alpha_{k}\right\}$ and $\left\{\beta_{k}\right\}$ are, respectively, an independently, identically distributed (i.i.d.)Rayleigh random variable with unit second moment and an i.i.d. uniform random variable over $[0,2 \pi)$. The received signal is expressed as

$$
\begin{aligned}
r(t) & =\sum_{k=1}^{K} \operatorname{Re}\left\{\zeta_{k} \sqrt{P_{k}} \sum_{j=-\infty}^{\infty} d_{k}(j) c_{k}(t-j T) e^{j\left(\omega_{0} t+\theta_{k}\right)}\right\}+w(t) \\
& =\sum_{k=1}^{K} \alpha_{k} \sqrt{P_{k}} \sum_{j=-\infty}^{\infty} d_{k}(j) c_{k}(t-j T) \cos \left(\omega_{0} t+\theta_{k}^{\prime}\right)+w(t)
\end{aligned}
$$




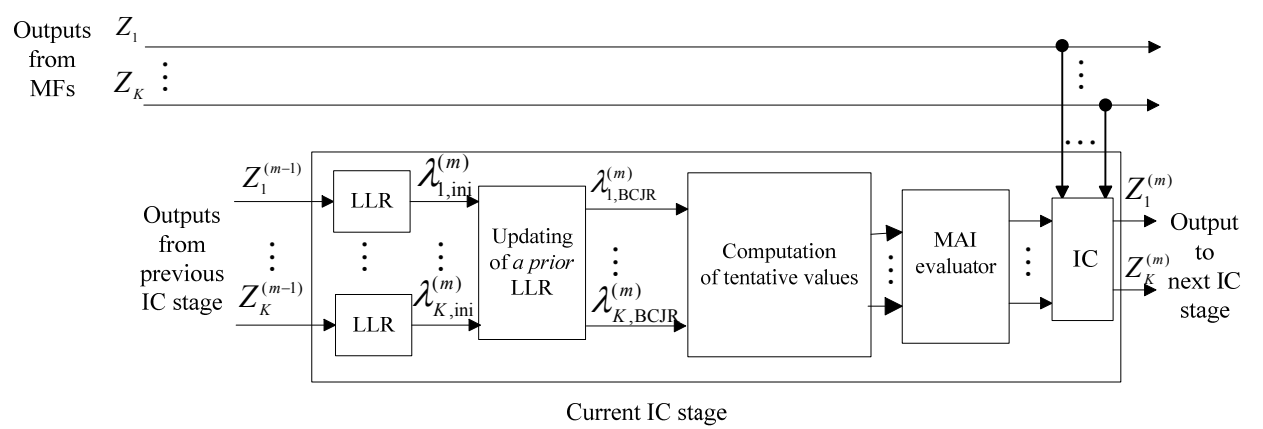

FIGURE I. THE $m$-th IC STAGE OF THE LOW-COMPLEXITY JOINT PIC/DECODING FOR CONVOLUTIONALLY CODED CDMA

where $\omega_{0}$ is carrier frequency, $\left\{\theta_{k}\right\}$ are random variables with a uniform distribution over $[0,2 \pi)$, and $\theta_{k}^{\prime}=\theta_{k}+\beta_{k}, T$ is coded symbol duration, $P_{k}$ and $K$ are the $k$-th user's transmitted power and the user number, respectively. The noise $w(t)$ is white Gaussian noise with a zero mean and two-sided power spectral density of $\eta_{0} / 2, d_{k}(j)$ denotes the $j$-th convolutionally coded symbol of user $k$, $c_{k}(t)=\sum_{l=0}^{N-1} c_{k}(l) p_{T_{c}}\left(t-l T_{c}\right), c_{k}(l) \in\{-1,1\}, N=T / T_{c}$, and

$$
p_{T_{c}}(t)=\left\{\begin{array}{ll}
1, & 0 \leq t<T_{c} \\
0, & \text { otherwise }
\end{array} .\right.
$$

Figure I illustrates the $m$-th IC stage of the proposed joint PIC/ decoding for convolutionally coded CDMA. In the proposed joint $\mathrm{PIC/}$ decoding, interference cancellation (IC) and updating of a priori LLR are associated with each other at at each IC stage.

Perform band-pass filtering, coherent demodulation and low-pass filtering (LPF) over the received signal, respectively, and we obtain the effective base-band signal as

$$
y_{k}(t)=\sum_{k^{\prime}=1}^{K} \sum_{j=-\infty}^{\infty} \alpha_{k^{\prime}} \sqrt{P_{k^{\prime}}} b_{k^{\prime}}(j) \sum_{n=0}^{N-1} c_{k^{\prime}}^{(j+n)} x\left(t-j T-n T_{c}\right) \cos \left(\theta_{k^{\prime}}^{\prime}-\theta_{k}^{\prime}\right)+w_{k}(t)
$$

and $w_{k}(t)=\operatorname{LPF}\left\{w(t) \sqrt{2} \cos \left(\omega_{0} t+\theta_{k}\right)\right\}, x(t)$ is the inverse Fourier transform of $|H(f)|^{2}$.

Perform despreading over $y_{k}(t)$ and use (3), and we have the correlation output as

$$
Z_{k}(i)=\sqrt{P_{k}} \alpha_{k} d_{k}(i)+I_{k}(i)+w_{k}(i)
$$

for coded symbol $i$ of user $k$, where $I_{k}(i)=\sum_{k^{\prime}=1, k^{\prime} \neq k}^{K} \frac{\alpha_{k^{\prime}} \sqrt{P_{k^{\prime}}}}{N} \cos \left(\theta_{k^{\prime}}^{\prime}-\theta_{k}^{\prime}\right) d_{k^{\prime}}(i) \rho_{k^{\prime}, k}$ and $\rho_{k^{\prime}, k}=\int_{0}^{T} c_{k}(t) c_{k^{\prime}}(t) d t$ is cross-correlation coefficient between user $k^{\prime}$ and user $k$ spreading sequences.
In the following, we analyze the proposed PIC/decoding with $M$ IC stages. Each IC stage has the same structure. With no loss of generality, we only give the description of the $m$-th IC stage.

\section{A. Description of the m-th IC Stage}

The $\left\{Z_{k}^{(m-1)}(i)\right\}$, which are the interference-cancelled outputs from the ( $m$-1)-th IC stage, are sent to the $m$-th IC stage. With conditionally Gaussian distribution [6] of $Z_{k}^{(m-1)}(i)$, we compute the mean and variance of $Z_{k}^{(m-1)}(i)$, respectively, as

$$
\mathrm{E}\left[Z_{k}^{(m-1)}(i)\right]=\sqrt{P_{k}} \alpha_{k} d_{k}(i)
$$

$$
\begin{gathered}
\sigma_{k}^{(m-1)}(i)=\operatorname{Var}\left[Z_{k}^{(m-1)}(i) \mid \alpha_{k}\right] \\
=\sum_{k^{\prime}=1, k^{\prime} \neq k}^{K} P_{k^{\prime}} \rho_{k^{\prime}, k}^{2} / 2 N^{2}-\sum_{k^{\prime}=1, k^{\prime} \neq k} P_{k^{\prime}} \rho_{k^{\prime}, k}^{2}\left[\widetilde{d}_{k^{\prime}}^{(m-1)}(i)\right]^{2} / 2 N^{2}+\eta_{0} / 2 N
\end{gathered}
$$

where $\widetilde{d}_{k}^{(m-1)}(i)$ is the tentative value for the $i$-th symbol of user $k$.

We define the initial a priori LLR of the current IC stage as

$$
\lambda_{k, \text { ini }}^{(m)}\left(d_{k}^{(m-1)}(i)\right)=\operatorname{In} \frac{\mathrm{P}\left(Z_{k}^{(m-1)}(i) \mid d_{k}(i)=+1\right)}{\mathrm{P}\left(Z_{k}^{(m-1)}(i) \mid d_{k}(i)=-1\right)}
$$

where

$$
\mathrm{P}\left(Z_{k}^{(m-1)}(i) \mid d_{k}(i)=+1\right)=\mathrm{A} \exp \left(-\left(Z_{k}^{(m-1)}(i)-1\right)^{2} /\left(2\left[\sigma_{k}^{(m-1)}(i)\right]^{2}\right)\right)
$$

$$
\mathrm{P}\left(Z_{k}^{(m-1)}(i) \mid d_{k}(i)=-1\right)=\mathrm{A} \exp \left(-\left(Z_{k}^{(m-1)}(i)+1\right)^{2} /\left(2\left[\sigma_{k}^{(m-1)}(i)\right]^{2}\right)\right)
$$

are the a priori probabilities of $d_{k}(i)=+1$ and $d_{k}(i)=-1$, respectively, where $\mathrm{A}=1 /\left[\sigma_{k}^{(m-1)}(i)\right]^{2}$. using (6) to (9), we obtain 


$$
\lambda_{k, \text { ini }}^{(m)}\left(d_{k}(i)\right)=\operatorname{In} \frac{\mathrm{P}\left(Z_{k}^{(m-1)}(i) \mid d_{k}(i)=+1\right)}{\mathrm{P}\left(Z_{k}^{(m-1)}(i) \mid d_{k}(i)=-1\right)}=\frac{2 \mu_{k}}{\left[\sigma_{k}^{(m-1)}(i)\right]^{2}} Z_{k}^{(m-1)}(i)
$$

where $\mu_{k}=\mathrm{E}\left[Z_{k}^{(m-1)}(i) d_{k}(i)\right]=\sqrt{P_{k}}$, and $\lambda_{k, \text { ini }}^{(m)}\left(d_{k}(i)\right)$, which is obtained from the $m$-th IC stage, is initial a priori LLR of $d_{k}(i)$.

We rewrite $d_{k}(i)$ as $d_{k, p}^{q}$ i.e., $d_{k}(i)=d_{k, p}^{q}$. By BCJR algorithm [7], initial a priori LLR is recursively updated. From [8-9], this updated a priori LLR is expressed as

$$
\begin{aligned}
& \lambda_{k, \mathrm{BCCR}}^{(m)}\left(d_{k}(i)\right)=\lambda_{k, \mathrm{BCCR}}^{(m)}\left(d_{k, p}^{q}\right) \\
& =\log \frac{\sum_{s_{q}^{\prime}} \alpha_{p-1}\left(s^{\prime}\right) \beta_{p}(s) \prod_{q^{\prime} \neq q} \mathrm{P}^{(m)}\left[d_{k, p}^{q^{\prime}}\left(s^{\prime}, s\right)\right]}{\sum_{s_{q}} \alpha_{p-1}\left(s^{\prime}\right) \beta_{p}(s) \coprod_{q^{\prime} \neq q} \mathrm{P}^{(m)}\left[d_{k, p}^{q^{\prime}}\left(s^{\prime}, s\right)\right]}
\end{aligned}
$$

where

$$
\begin{aligned}
& \alpha_{p}(s)=\sum_{s^{\prime}} \alpha_{p-1}\left(s^{\prime}\right) \prod_{q^{\prime}=1}^{n} \mathrm{P}^{(m)}\left[d_{k, p}^{q^{\prime}}\left(s^{\prime}, s\right)\right], \quad p=1,2, \cdots, \tau \\
& \beta_{p}(s)=\sum_{s^{\prime}} \beta_{p+1}\left(s^{\prime}\right) \prod_{q^{\prime}=1}^{n} \mathrm{P}^{(m)}\left[d_{k, p+1}^{q^{\prime}}\left(s^{\prime}, s\right)\right], \quad p=\tau-1, \tau-2, \cdots, 0
\end{aligned}
$$

with boundary condition $\alpha_{0}(0)=1, \alpha_{0}(s \neq 0)=0$; and $\beta_{\tau}(0)=1, \beta_{\tau}(s \neq 0)=0 . \quad \mathrm{P}^{(m)}\left[d_{k, p}^{q^{\prime}}\left(s^{\prime}, s\right)\right]$ is the transition probability from $s^{\prime}$ to $s$. The (11), (12) and (13) are detailed in [8].

$$
\mathrm{P}^{(m)}\left[d_{k, p}^{q^{\prime}}\left(s^{\prime}, s\right)\right]=\frac{1}{2}\left[1+d_{k, p}^{q^{\prime}}\left(s^{\prime}, s\right) \tanh \left(\frac{1}{2} \lambda_{k, \mathrm{nin}}^{(m)}\left(d_{k, p}^{q^{\prime}}\right)\right)\right]
$$

The computation of (14) involves tangent function, which increases the computation complexity. But if we use

$$
\tanh \left(\frac{1}{2} \lambda_{k, \mathrm{ini}}^{(m)}\left(b_{k, p}^{q^{\prime}}\right)\right) \approx\left\{\begin{array}{cc}
\lambda_{k, \text { ini }}^{(m)}\left(d_{k, p}^{q^{\prime}}\right) / 2, & \left(\lambda_{k, \mathrm{in}}^{(m)}\left(d_{k, p}^{q^{\prime}}\right) \leq 2\right) \\
\operatorname{sgn}\left(\lambda_{k, \mathrm{ini}}^{(m)}\left(d_{k, p}^{q^{\prime}}\right)\right), & \left(\lambda_{k, \mathrm{ini}}^{(m)}\left(d_{k, p}^{q^{\prime}}\right)>2\right)
\end{array}\right.
$$

the computation of (14) can be greatly simplified.

Tentative value for each coded symbol is given as

$$
\widetilde{d}_{k}^{(m)}(i)=\mathrm{P}\left(Z_{k}^{(m-1)}(i) \mid d_{k}(i)=+1\right)-\mathrm{P}\left(Z_{k}^{(m-1)}(i) \mid d_{k}(i)=-1\right)
$$

In terms of updated a priori LLR, Using (7), (8), (9) and (11), with some manipulations, we can obtain

$$
\tilde{d}_{k}^{(m)}(i)=\tanh \left(\lambda_{k, \mathrm{BCJR}}^{(m)}\left(d_{k}(i) / 2\right)\right)
$$

For the same approximation as (15), the computation of (17) can be further simplified as

$$
\widetilde{d}_{k}^{(m)}(i) \approx\left\{\begin{array}{cc}
\lambda_{k \mathrm{BCIR}}^{(m)}\left(d_{k}(i)\right) / 2, & \left(\lambda_{k, \mathrm{BCIR}}^{(m)}\left(d_{k}(i)\right) \leq 2\right) \\
\operatorname{sgn}\left(\lambda_{k, \mathrm{BCIR}}^{(m)}\left(d_{k}(i)\right)\right), & \left(\lambda_{k, \mathrm{BCJR}}^{m}\left(d_{k}(i)\right)>2\right)
\end{array}\right.
$$

which is a linear expression.

If we substitute $\lambda_{k, \mathrm{BCJR}}^{(m)}\left(d_{k}(i)\right)$ by the sum of initial a priori LLR and updated a priori LLR, (18) becomes

$$
\widetilde{d}_{k}^{(m)}(i) \approx\left\{\begin{array}{cc}
\lambda_{k, \text { sum }}^{(m)}\left(d_{k}(i)\right) / 2, & \left(\lambda_{k, \text { sum }}^{(m)}\left(d_{k}(i)\right) \leq 2\right) \\
\operatorname{sgn}\left(\lambda_{k, \text { sum }}^{(m)}\left(d_{k}(i)\right)\right), & \left(\lambda_{k, \text { sum }}^{(m)}\left(d_{k}(i)\right)>2\right)
\end{array}\right.
$$

where $\lambda_{k, \text { sum }}^{(m)}\left(d_{k}(i)\right)=\lambda_{k, \text { ini }}^{(m)}\left(d_{k}(i)\right)+\lambda_{k, \mathrm{BCJR}}^{(m)}\left(d_{k}(i)\right)$.

The tentative values from (18) or (19), together with other known information such as all users' spreading sequences, are used to reconstruct MAI, the estimated MAI is subtracted from MF outputs, and we have $Z_{k}^{(m)}(i)=Z_{k}(i)-\widetilde{I}_{k}^{(m)}(i)$.

For the last IC stage, a hard decision is made on $Z_{k}^{(M)}(i)$, and then input to the single Viterbi decoder for decoding.

\section{SimULATION RESUlTS}

This section simulates the proposed joint PIC/decoding. In the simulations, we choose such parameters as: (1) the user number $\mathrm{K}=8$; $(2)$ the rate $\mathrm{R}=1 / 2$ and constraint length $v=3$

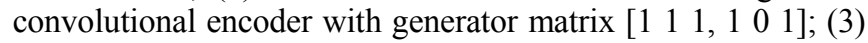
the randomly generated short spreading sequences with spreading factor $N=13$; (4) the data frame length 128 .

In the following, for convenience of description, we call the joint PIC/decoding expressed by (17), (18) and (19) as IdealJPIC/decoding, Linear JPIC/decoding with a priori LLR and Linear JPIC/decoding with a posteriori LLR, respectively.

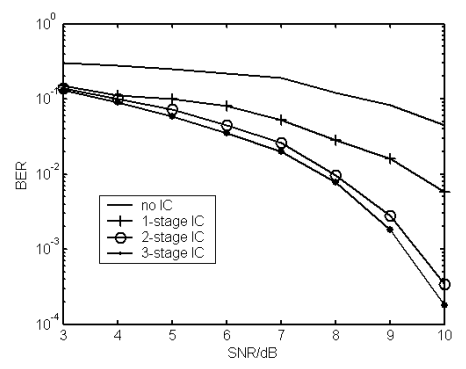

FIGURE II. PERFORMANCE OF IDEAL-JPIC/DECODING

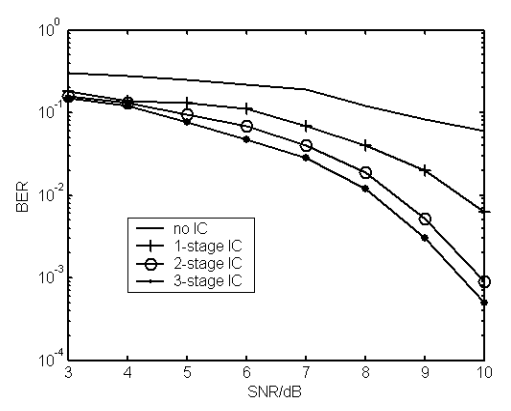

FIGURE III. PERFORMANCE OF LINEAR-JPIC/DECODING WITH $A$ POSTERIORI LLR 
Figure II, Figure III and Figure IV show the performance of Ideal-JPIC/decoding, Linear-JPIC/decoding with a posteriori LLR and Linear-JPIC/decoding with a priori LLR, respectively. Performance is compared for 1,2 and 3 IC stages. It is clear from three figures that three PIC/decodings has common points in performance change: as the number of IC stage increases, performance of the bit error error improves at the same signalto-noise ratio (SNR), and compared to conventional receiver with no IC, the first 2 IC stages brings the biggest performance improvement, but the performance improvement by the third stage IC is gradually less obvious. For example, in Figure II, at SNR of $8 \mathrm{~dB}$, the bit error rate for the first, the second stages IC and the third IC stage is about $3 \times 10^{-2}$ and $9 \times 10^{-3}, 8 \times 10^{-3}$, respectively, which are far below the BER of the conventional receiver with no IC, which is $1 \times 10^{-1}$. Apparently, the third stage IC only brings slight performance improvement, compared to the second stage IC.

Figure V and Figure VI compare the performance of bit error rate of three joint PIC/decodings in case of two and three IC stages, respectively. It can be seen that whether with two IC stages or with three IC stages, Ideal-JPIC/decoding exibits the best performance, Linear-JPIC/decoding with a posteriori LLR acquires a performance close to Ideal-JPIC/decoding, but far supeior to Linear-JPIC/decoding with a priori LLR at the same SNR. For example, in Figure VI, at SNR of 9dB, the BERs for Ideal-JPIC/decoding, Linear-JPIC/decoding with a posteriori LLR and Linear-JPIC/decoding with a priori LLR are about 2 $\times 10^{-3}$ and $4 \times 10^{-3}, 7 \times 10^{-3}$, respectively.

Figure VII compares the performance of the proposed algorithm, separate PIC and decoding (SPIC/decoding), and conventional joint PIC and decoding (JPIC/decoding) with the same three IC stages. As one of three proposed algorithms, Linear-JPIC/decoding with a priori LLR is chosen to compare with the other alorithms in performance. Obviously, the proposed algorithm exibits the dominant performance advantage over the other two conventional algorithms. For example, at SNR of $9 \mathrm{~dB}$, the BERs for the proposed algorithm, $\mathrm{JPIC} /$ decoding and SPIC/decoding are about $3 \times 10^{-3}$ and $1 \times 10^{-2}$, $2 \times 10^{-2}$, respectively. It is also seen that among three algorithms, the SPIC/decoding has the worst performance.

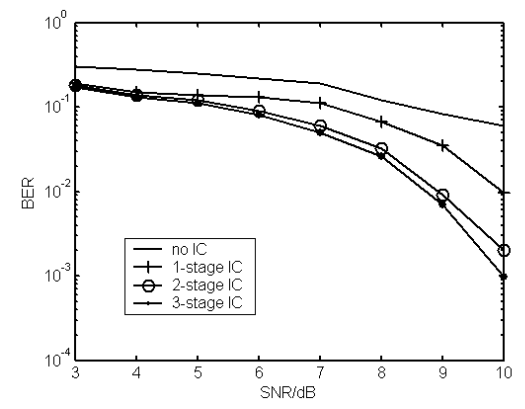

FIGURE IV. PERFORMANCE OF LINEAR-JPIC/DECODING WITH $A$ PRIORI LLR

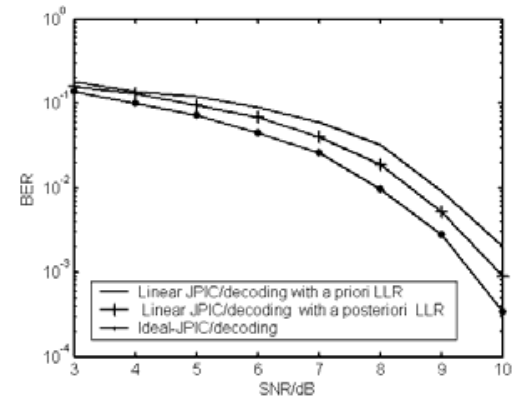

FIGURE V. PERFORMANCE COMPARISON OF THREE JOINT PIC/DECODINGS WITH TWO IC STAGES

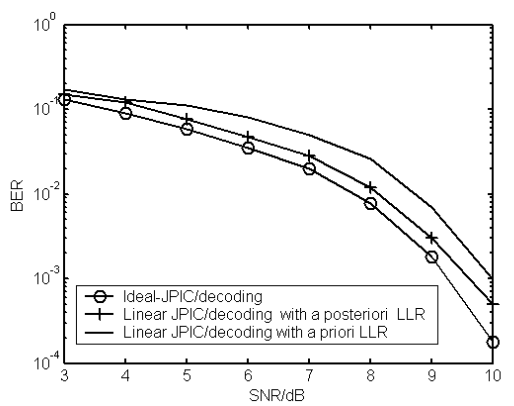

FIGURE VI. PERFORMANCE COMPARISON OF THREE JOINT PIC/DECODINGS WITH THREE IC STAGES

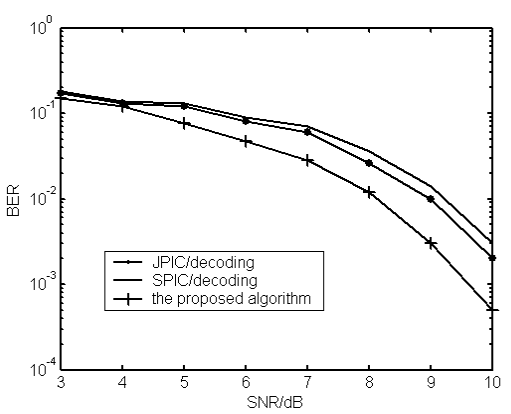

FIGURE VII. PERFORMANCE COMPARISON OF THE PROPOSED ALGORITHM, SPIC/DECODING AND CONVENTIONAL JPIC/DECODING

\section{CONCLUSIONS}

In this paper, we propose a new joint PIC/decoding for convolutionally coded DS-CDMA systems. At each interference cancellation stage, initial a priori LLR is computed by the output from the previous IC stage, and input to convolutional decoder. The decoder utilizes BCJR algorithm to update initial a priori LLR. Initial a priori LLR and updated a priori LLR are summed to obtain the tentative values of coded symbols linearly, which are used to estimated MAI. The proposed joint PIC/decoding is numerically simulated and compared with the other joint PIC/decoding, conventional $\mathrm{PIC} /$ decoding and MF receiver in performance, respectively. According to the simulation results, we draw the main conclusions including: (1) Like conventional PIC and decoding algorithms, the proposed algorithms exhibit better performance with the increase in the number of the interference cancellation 
(IC) stages, but up to three IC stages, the performance improvement is getting less obvious; (2)The proposed joint $\mathrm{PIC} /$ decoding acquires the performance close to the ideal joint $\mathrm{PIC} /$ decoding if the tentative decisions of coded symbols are linearly estimated by the sum of initial a priori log-likelihood rate (LLR) and updated a priori LLR, while a significant performance degradation will evitably occur if the tentative decisions of coded symbols are linearly computed by the updated a priori LLR only. We also compare the proposed PIC/decoding with matched-filter (MF) receiver and conventional PIC/decoding algorithms, respectively. The proposed joint PIC/decoding dominantly outperforms the MF receiver and conventional PIC/decodings in performance.

\section{REFERENCES}

[1] S.Moshavi, "Multi-user detection for DS-CDMA communications," IEEE Commun. Mag., vol. 34, pp.124 137, Oct.1996.

[2] L.Fang and L.B.Milstein, "Successive interference cancellation in multicarrier DS/ CDMA,” IEEE Trans. Commun., vol.48, pp.1530 1540, Sept. 2000.

[3] G.X. Xu, "Turbo IC/decoding for multi-carrier DS-CDMA," in Proc.of ICCC2013, Wuhan, pp.74-77, Nov.2013.

[4] A. Nahler, R. Irmer and G. Fettweis, "Reduced and differential parallel interference cancellation for CDMA systems," IEEE J. Select. Areas Commun., vol.20, pp.237-247, Feb. 2002.

[5] Y. Sanada and Q. Wang, "A co-channel interference cancellation technique using orthogonal convolutional codes," IEEE Trans. Commun., vol.44, pp.549-556, May 1996.

[6] D. Divsalar, M.K. Simon and D. Raphaeli, "Improved parallel interference cancellation for CDMA," IEEE Trans.Commun., vol.46, pp.258-268, Feb. 1998.

[7] L.R. Bahl, J. Cocke, F. Jelinek and J. Raviv, "Optimal decoding of linear codes for minimizing symbol error rate," IEEE Trans. Commun., vol. IT20, pp.284-287, March 1976.

[8] X. Wang and H. Vincent, "Iterative (Turbo) soft interference cancellation and decoding for coded CDMA," IEEE Trans. Commun., vol.47, pp.1046-1061, July 1999.

[9] L. C. Gan and G.X.Xu, "Turbo interference cancellation/decoding for cnvolutionally coded multi-carrier DS-CDMA," Chinese Journal of Electronics, vol.14,pp.309-313, April 2005 